\title{
ON THE PRODUCT OF SINGULAR SYMMETRIC MATRICES
}

\section{LOUIS BRAND}

The following theorem has been conjectured and used in statistical applications, but, so far as known, has not been proved.

THEOREM. $A$ and $B$ are real $n \times n$ symmetric matrices with $\lambda_{1}, \cdots, \lambda_{r}$, $0, \cdots, 0$ and $0, \cdots, 0, \lambda_{r+1}, \cdots, \lambda_{n}\left(\lambda_{1} \neq 0\right)$ as eigenvalues respectively. If $A+B$ has $\lambda_{1}, \lambda_{2}, \cdots, \lambda_{n}$ as eigenvalues, then $A B=0$.

Proof. If $C$ is a symmetric matrix having $\lambda_{1}, \cdots, \lambda_{n}$ as eigenvalues, then $C$ can be put in the dyadic form [1]

$$
C=\lambda_{1} c_{1} c^{1}+\cdots+\lambda_{n} c_{n} c^{n}, \quad c^{i} c_{j}=\delta_{j}^{i},
$$

where the $c_{i}$ are unit column vectors that form an orthonormal set and $c^{i}$ is the row vector transpose of $c_{i}$. The $n$ dyads $c_{i} c^{i}$ are $n \times n$ matrices of rank 1 , and are precisely the $n$ idempotents of $C$, whereas the products $c^{i} c_{j}$ are $1 \times 1$ matrices, the numbers $\delta_{j}^{i}$.

Thus $A$ and $B$ have the dyadic form

$$
\begin{aligned}
& A=\lambda_{1} a_{1} a^{1}+\cdots+\lambda_{r} a_{r} a^{r}, \quad a^{i} a_{j}=\delta_{j}^{i}, \\
& B=\lambda_{r+1} b_{r+1} b^{r+1}+\cdots+\lambda_{n} b_{n} b^{n}, \quad b^{i} b_{j}=\delta_{j}^{i} .
\end{aligned}
$$

Any matrix in dyadic form can be factored:

$$
M=p_{1} q^{1}+\cdots+p_{n} q^{n}=P Q
$$

where $P$ has $p_{1}, \cdots, p_{n}$ as columns, $Q$ has $q^{1}, \cdots, q^{n}$ as rows; and $\operatorname{det} M=\operatorname{det} P \cdot \operatorname{det} Q$. Therefore $\operatorname{det}(A+B)=\lambda_{1} \lambda_{2} \cdots \lambda_{n} \Delta^{2}$ where $\Delta$ is the determinant whose rows are the unit vectors $a^{1}, \cdots, a^{r}$, $b^{r+1}, \cdots, b^{n}$. But when the symmetric matrix $A+B$ is put in the form (1), $\operatorname{det}(A+B)=\lambda_{1} \lambda_{2} \cdots \lambda_{n}$; and since $\lambda_{i} \neq 0$, this implies $\Delta^{2}=1$. By the Hadamard determinant theorem,

$$
\Delta^{2} \leqq\left|a^{1}\right|^{2} \cdots\left|a^{r}\right|^{2}\left|b^{r+1}\right|^{2} \cdots\left|b^{n}\right|^{2}=1
$$

with equality when, and only when, the vectors are mutually orthogonal; thus $a^{i} b_{j}=0(1=i, \cdots, r ; j=r+1, \cdots, n)$ and $A B=0$.

\section{REFERENCES}

1. M. Pease, Methods of matrix algebra, Academic Press, New York, 1965.

UNIVERSITY OF HOUSTON

Received by the editors December 10, 1968. 